\title{
SPECTRALLY BOUNDED GENERALIZED INNER DERIVATIONS
}

\author{
RAÚL CURTO AND MARTIN MATHIEU
}

(Communicated by Eric Bedford)

\begin{abstract}
We characterize the generalized inner derivations on a unital Banach algebra which are spectrally bounded. In particular, a simplified argument for the recent result due to Brešar that every spectrally bounded inner derivation maps into the radical is given.
\end{abstract}

In [3], a linear mapping $T$ on a complex unital Banach algebra $A$ was defined to be spectrally bounded if $r(T x) \leq M r(x)$ for all $x \in A$ and some $M \geq 0$, where $r(\cdot)$ denotes the spectral radius. This concept arises in connection with the non-commutative Singer-Wermer conjecture stating that the image $\delta A$ of any derivation $\delta$ on $A$ is contained in the Jacobson $\operatorname{radical} \operatorname{rad}(A)$ if and only if $x \delta x-(\delta x) x \in \operatorname{rad}(A)$ for all $x \in A$. Although some progress on this conjecture has been made lately (see [6] and the references in [3]), it is still open and seems hard to verify.

A related, also open, conjecture is the following: The image $\delta A$ of a derivation $\delta$ on $A$ is contained in $\operatorname{rad}(A)$ if and only if $\delta$ is spectrally bounded. This was recently affirmed for inner derivations by Brešar [2]. His argument rests in an essential way on two results due to Pták [5], in particular on [5, Proposition 2.1 stating that a spectrally bounded inner derivation $\delta$ has the property that $\delta^{2} A \subseteq \mathscr{Q}(A)$, the set of quasinilpotent elements of $A$. In an earlier paper, however, Pták obtained a number of equivalent conditions for the image of an inner derivation to lie in the radical. For our purposes, the following suffices.

Theorem A [4, Proposition 2.1]. Let $A$ be a unital Banach algebra and $a \in A$. The following conditions are equivalent.

(a) $a x-x a \in \operatorname{rad}(A)$ for all $x \in A$.

(b) $r(a x) \leq M r(x)$ for all $x \in A$ and some $M \geq 0$.

While $(a) \Rightarrow(b)$ is immediate, the implication $(b) \Rightarrow(a)$ is a consequence of the subharmonicity of the spectral radius. (But see the note at the end of this paper.)

Let $L_{a}$ and $R_{a}$ denote the left and right multiplications by $a \in A$, respectively. Combining Theorem A with Brešar's result we have that $L_{a}-R_{a}$ is

Received by the editors July 8, 1993 and, in revised form, November 28, 1993; presented by the first-named author at the Special Session on Nonselfadjoint Operator Algebras, 886th Regional Meeting of the AMS, College Station, Texas, October 22, 1993.

1991 Mathematics Subject Classification. Primary 46H99; Secondary 47B47.

The first-named author's research was partially supported by an NSF grant. 
spectrally bounded if and only if $L_{a}$ (equivalently $R_{a}$ ) is spectrally bounded. The main result of the present paper (Theorem B) extends this appropriately to the situation of generalized inner derivations $L_{a}-R_{b}, a, b \in A$. In fact, our proof is more direct than Brešar's argument in the case $a=b$ and uses only Theorem A, bypassing the results in [5] (which are not available for $L_{a}-R_{b}$ ). As a result, Brešar's theorem is an immediate consequence of Theorems $A$ and B.

Little seems to be known about the class of spectrally bounded operators in general. Apart from the obvious ones, e.g., unital homomorphisms, there are not many examples. We therefore precede our results by compiling a few basic facts. We shall use the following additional terminology. A linear mapping $T: A \rightarrow A$ is said to be spectrally infinitesimal if $r(T x)=0$ for all $x \in A$, i.e., $T A \subseteq \mathscr{Q}(A)$, and is called spectrally isometric if $r(T x)=r(x)$ for all $x \in A$, i.e, $T$ is spectral-radius preserving.

Facts. 1. The set $S B(A)$ of all spectrally bounded operators on $A$ is a multiplicative semigroup with identity. However, the sum of two spectrally bounded operators will in general not be spectrally bounded; a condition assuring this is that their images commute modulo $\operatorname{rad}(A)$.

2. The canonical epimorphism $\rho: A \rightarrow A / \operatorname{rad}(A)$ is a spectral isometry. Thus, if $T \in S B(A)$ and $S: A \rightarrow A$ is linear with $S A \subseteq \operatorname{rad}(A)$, then $T+S \in$ $S B(A)$.

3. For each $a \in A, L_{a}$ (equivalently, $R_{a}$ ) is spectrally infinitesimal if and only if $a \in \operatorname{rad}(A)$ [1, Theorem 3.1.3].

4. A spectrally infinitesimal derivation on $A$ maps into the radical. This can be found in various places (see, e.g., [3]).

Whenever $a, b \in A$ are such that $L_{a}$ and $R_{b}$ are spectrally bounded, then the product $L_{a} R_{b}$ is spectrally bounded; the converse is not true in general. For the difference, however, the equivalence holds.

Theorem B. Let $A$ be a unital Banach algebra and $a, b \in A$. The following conditions are equivalent.

(a) $L_{a}-R_{b}$ is spectrally bounded.

(b) Both $L_{a}$ and $R_{b}$ are spectrally bounded.

Proof. (a) $\Rightarrow$ (b) Note at first that it suffices to show that $R_{b}$ is spectrally bounded. By Pták's result, $b$ is then central modulo $\operatorname{rad}(A)$ wherefore

$$
\begin{aligned}
r(a x-x b) & =r(\rho(a) \rho(x)-\rho(x) \rho(b)) \\
& =r((\rho(a)-\rho(b)) \rho(x)) \\
& =r(\rho(x)(\rho(a)-\rho(b))) \\
& =r(\rho(b) \rho(x)-\rho(x) \rho(a)) \\
& =r(b x-x a) \quad \text { for all } x \in A .
\end{aligned}
$$

Thus, $L_{b}-R_{a}$ is spectrally bounded as well, which implies that $R_{a}$, and hence $L_{a}$, is spectrally bounded.

Suppose that $R_{b}$ is not spectrally bounded. Then, by Theorem $\mathrm{A}$, there is an irreducible representation $\pi$ of $A$ such that $\pi(b) \notin \mathbb{C} 1$. Take a vector $\xi$ in the representation space such that $\{\xi, \pi(b) \xi\}$ is linearly independent. By Jacobson's density theorem [1, Theorem 4.2.5], we can find $x$ and $u$ in $A$ 
such that

$$
\pi(x) \xi=0, \quad \pi(x) \pi(b) \xi=\xi, \quad \pi(u) \pi(b) \xi=\pi(b) \xi, \quad \text { and } \quad \pi(u) \xi=-n \xi,
$$

where $n \in \mathbb{N}$ is arbitrary. In fact, by Sinclair's theorem [1, Corollary 4.2.6], we may even assume that $u$ is invertible. Then

$$
\left(\pi(a) \pi(u) \pi(x) \pi(u)^{-1}-\pi(u) \pi(x) \pi(u)^{-1} \pi(b)\right) \xi=n \xi,
$$

whence $r\left(a u x u^{-1}-u x u^{-1} b\right) \geq n$.

However, this violates the estimate

$$
r\left(a u x u^{-1}-u x u^{-1} b\right) \leq \operatorname{Mr}\left(u x u^{-1}\right)=\operatorname{Mr}(x)
$$

for some constant $M \geq 0$. As a result, $R_{b}$ has to be spectrally bounded.

(b) $\Rightarrow$ (a) If $L_{a}$ is spectrally bounded, so is $R_{a}$; whence, by assumption and Theorem $\mathrm{A}, R_{a-b}$ is spectrally bounded. In addition, $L_{a}-R_{a}$ maps into the radical. Hence, by Fact 2 ,

$$
L_{a}-R_{b}=R_{a-b}+L_{a}-R_{a}
$$

is spectrally bounded.

Corollary [2, Theorem]. Every spectrally bounded inner derivation maps into the radical.

Proof. This is immediate from Theorems B and A.

Unlike inner derivations, spectrally bounded generalized inner derivations need not map into the radical. Indeed, those that do differ from inner derivations only by an additive perturbation consisting of a multiplication by an element in the radical.

Proposition 1. The following conditions on $a, b \in A$ are equivalent.

(a) $L_{a}-R_{b}$ maps into $\operatorname{rad}(A)$.

(b) $\rho(a)=\rho(b)$ is central.

Proof. (a) $\Rightarrow$ (b) If $\left(L_{a}-R_{b}\right) A \subseteq \operatorname{rad}(A)$, then $a-b \in \operatorname{rad}(A)$ and so $R_{a-b}$ maps into $\operatorname{rad}(A)$. Thus,

$$
L_{a}-R_{a}=L_{a}-R_{b}-R_{a-b}
$$

maps into $\operatorname{rad}(A)$ as well. Consequently, $\rho(a)=\rho(b)$ is central in $A / \operatorname{rad}(A)$. Clearly, this argument can be reversed to obtain (b) $\Rightarrow(\mathrm{a})$.

On the other hand, Fact 4 takes over to generalized inner derivations.

Proposition 2. Let $a, b \in A$ be such that $L_{a}-R_{b}$ is spectrally infinitesimal. Then $\left(L_{a}-R_{b}\right) A \subseteq \operatorname{rad}(A)$.

Proof. By Theorem B, the assumption implies that $L_{a}$ is spectrally bounded; whence $L_{a}-R_{a}$ maps into $\operatorname{rad}(A)$ by Theorem A. Moreover, the first part of the proof of Theorem B shows that $r((a-b) x)=r(a x-x b)=0$ for all $x \in A$; hence, by Fact $3, a-b \in \operatorname{rad}(A)$ as well. Therefore,

$$
L_{a}-R_{b}=L_{a}-R_{a}+R_{a-b}
$$

maps into $\operatorname{rad}(A)$.

Note. We observe that the second part of the proof of $(\mathrm{a}) \Rightarrow(\mathrm{b})$ in Theorem B shows in fact that if $\left(L_{a}-R_{a}\right) A \nsubseteq \operatorname{rad}(A)$, i.e., $a$ is not central modulo 
$\operatorname{rad}(A)$, then $R_{a}$, and hence $L_{a}$, cannot be spectrally bounded. This gives a new independent argument for Theorem A, avoiding the subharmonicity of the spectral radius.

\section{ACKNOWLEDGMENT}

This paper was written while the second-named author enjoyed the hospitality of the University of Iowa.

\section{ADDED IN PROOF}

Building on the techniques of the present paper, Brešar together with the second-named author recently affirmed the general case, i.e., every spectrally bounded derivation maps into the radical [Derivations mapping into the radical, III, J. Funct. Anal. (to appear)], whence Theorem B also extends (appropriately) to non-inner spectrally bounded generalized derivations.

\section{REFERENCES}

1. B. Aupetit, A primer on spectral theory, Springer-Verlag, New York, 1991.

2. M. Brešar, Derivations decreasing the spectral radius, Arch. Math. (Basel) 61 (1993), 160-162.

3. M. Mathieu, Where to find the image of a derivation, Banach Center Publ. 30 (1994), 237-249.

4. V. Pták, Derivations, commutators and the radical, Manuscripta Math. 23 (1978), 355-362.

5. __ Commutators in Banach algebras, Proc. Edinburgh Math. Soc. 22 (1979), 207-211.

6. M. Thomas, Primitive ideals and derivations on non-commutative Banach algebras, Pacific J. Math. 159 (1993), 139-152.

Department of Mathematics, The University of Iowa, Iowa City, Iowa 52242

E-mail address: curto@math. uiowa.edu

Mathematisches Institut, Universität Tübingen, Auf der Morgenstelle 10, D-72076, TÜBINGEN, GermanY

Current address: Fachbereich 9 Mathematik, Universität des Saarlandes, D-66041 Saarbrücken, Germany

E-mail address: mm@math.uni-sb.de 